\title{
Evaluer la densité des ligneux par la méthode des distances ? Un estimateur
}

\author{
Ph. Daget ${ }^{1}$ A. Ickowicz ${ }^{2}$
}

Mots-clés

Pâturages - Mesure - Méthode Echantillonnage - Végétation - Densité Plante ligneuse - Zone tropicale.

\section{Résumé}

Les auteurs reprennent un estimateur de la densité à partir d'observations proches de celles des Plotless methods, en montrent le fonctionnement et l'applicabilité au cas des ligneux dans les savanes.

\section{INTRODUCTION}

La précédente note (6) a mis en évidence l'origine de la distorsion observée entre la densité calculée à partir des formules de Clarks et Evans (4) et la densité vraie correspondant au dénombrement réel des individus sur un hectare. Cependant, les mesures de densité restent très employées par les spécialistes de la faune sauvage, particulièrement par les ornithologues (7), ainsi que par des forestiers cherchant à évaluer le volume de matière combustible en sous-bois (9). De telles mesures peuvent aboutir à des valeurs réalistes par l'emploi d'estimateurs. Crain et coll. (8) ont émis une proposition qui a fait l'objet d'analyses mathématiques approfondies $(1,5)$. Nous en proposons ici une présentation pratique " débarrassée » des considérations théoriques que le lecteur intéressé pourra trouver dans les travaux cités ci-dessus.

\section{REALISATIO N DES MESU RES}

\section{Principe des observations}

Avant tout, il est nécessaire que les observations soient bien normalisées. On matérialise sur le terrain une «base » de longueur $\mathbf{L}$ (en général, $\mathrm{L}=100 \mathrm{~m}$, mais ce n'est pas indispensable). On fixe ensuite le nombre $\mathbf{N}$ de points de mesure sur la base $(\mathrm{N}=40$ est commode).

L'étape suivante consiste à mesurer, en face de chaque point et perpendiculairement à la ligne, la distance à la base du pied de l'individu le plus proche, soit d'un côté, soit de l'autre (figure 1).

1. Cirad-emvt/Cnrs, Campus international de Baillarguet, TA 30/F, 34398 Montpellier Cedex 5, France ; E-mail : philippe.daget@cirad.fr

2. Cirad-emvt/Isra-Lnerv, BP 2057, Dakar, Sénégal

E-mail : ickowicz@telecomplus.sn
Le fait que la distance soit mesurée au droit des points constitue la différence entre cette méthode et celle, originale, de Clark et Evans (4).

Enfin, toutes les distances $\mathbf{Z} \mathbf{j}$ supérieures à un seuil $\mathbf{W}$ fixé a prior (il est commode de prendre $\mathrm{W}=65 \mathrm{~m}$ ) sont éliminées ; on ajoute des observations de manière à en avoir exactement $\mathbf{n}$. Les calculs se font donc sur une distribution tronquée des $\mathrm{Zj}$.

En définitive, au retour du terrain, on a donc 40 mesures $\mathrm{Zj}$, toutes inférieures à $65 \mathrm{~m}$, à partir desquelles il faut évaluer la densité en nombre moyen d'individus à l'hectare.

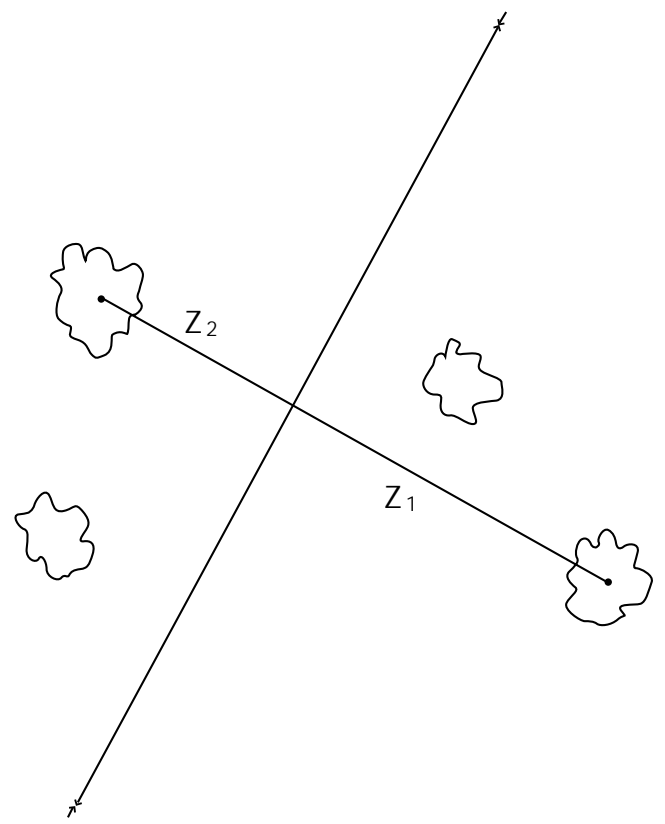

Figure 1 : dispositif d'observation sur le terrain ; comme $\mathrm{Z}_{2}<\mathrm{Z}_{1}$, la distance retenue est $\mathrm{Z}_{2}$. 


\section{Réalisation pratique}

De telles observations sont grandement facilitées par l'emploi d'un matériel d'arpenteur ou de géomètre : jalons colorés, viseurs, équerres d'arpenteur, rouleaux de fil et hectomètre à ruban... Mais on pourra suppléer les viseurs et l'équerre par une «boîte de visée » (figure 2 ).

Quel que soit le moyen utilisé, matériel d'arpenteur ou bricolé, on matérialise en premier lieu la «base », c'est-à-dire une ligne droite de longueur L par $\mathrm{N}$ jalons alignés au moyen de la boîte de visée.

Compte tenu des valeurs recommandées pour $\mathrm{L}$ et $\mathrm{N}$, on alignera 40 jalons séparés de 33 pas ; en effet, 33 pas de $0,75 \mathrm{~m}$ font $25 \mathrm{~m}$, cette distance reproduite 40 fois donne une longueur de $1000 \mathrm{~m}$.

Cela fait, la boîte de visée est positionnée à l'emplacement de chaque jalon pour déterminer l'individu le plus proche dans la visée perpendiculaire à la base. Pour cela, il faut viser le tronc des arbres (ou des palmiers) ou la souche des ligneux multicaules successivement d'un côté puis de l'autre et ne retenir que la plus faible des deux distances. Il est clair que deux personnes sont nécessaires pour aboutir à un résultat. Les opérations sont assez longues, mais moins que le dénombrement total du contenu d'un hectare de densité appréciable.

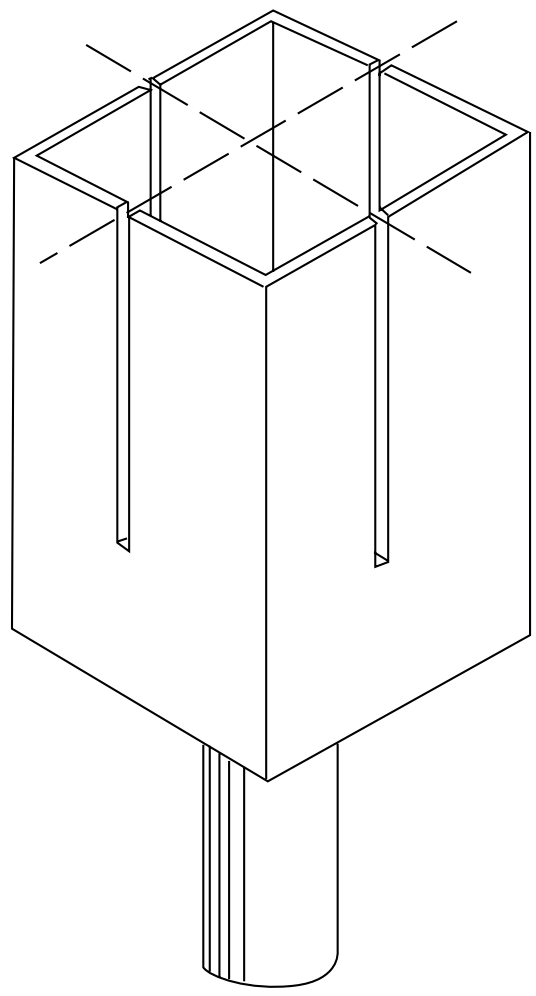

Figure 2 : pour construire une «boîte de visée », joindre par un trait de scie les milieux des côtés d'une boîte en bois de section carrée, un peu plus haute que large, sur les troisquarts de la hauteur et ébarber les bords des fentes ainsi faites. Marquer puis perforer le centre du fond de la boîte et la fixer par une vis sur un manche droit et rigide (un manche à balai par exemple). Cette boîte peut tenir lieu de viseur d'alignement et d'équerre optique. Les jalons pourront être remplacés par des individus munis d'une petite feuille de papier coloré placée dans une fente faite à l'une des extrémités.

\section{Au laboratoire}

\section{L'estimateur}

Crain et coll. (5) montrent que la densité D peut être approchée par:

$$
\mathrm{D}=\frac{\mathrm{nf}(0)}{2 \mathrm{~L}}
$$

expression dans laquelle :

$$
f(0)=\frac{1}{W}+\sum_{i=1}^{40} \frac{2}{W N} \sum_{j=1}^{3} \cos \frac{k \pi Z_{j}}{W}
$$

Dans cette formule, les cosinus s'introduisent par l'intermédiaire des séries de Fourier. L'expression peut paraître complexe, surtout que les angles sous le signe « cosinus « doivent être exprimés en radians, mais le petit programme ci-dessous montre, par sa brièveté, qu'il n'en est rien :

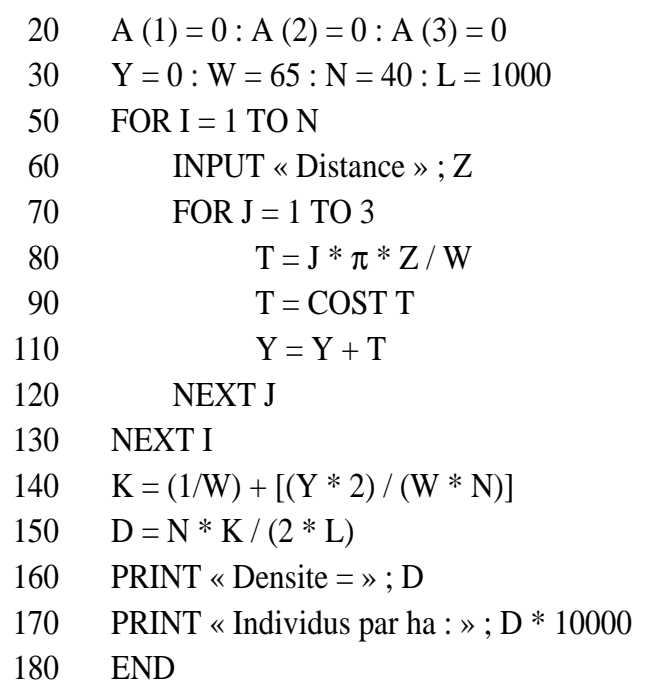

\section{Simulation}

Une observation

Elle est proposée par Crain (5) ; dans une population dont la densité vraie est de 5,04 par ha, les 40 distances mesurées sur $1 \mathrm{~km}$ sont :

$\begin{array}{rrrrr}24,10 & 10,98 & 8,30 & 48,56 & 48,05 \\ 32,92 & 43,20 & 27,61 & 32,68 & 17,48 \\ 8,53 & 27,26 & 25,25 & 23,56 & 14,99 \\ 36,76 & 14,27 & 16,25 & 24,78 & 37,84 \\ 2,14 & 61,88 & 59,69 & 44,42 & 5,32 \\ 37,61 & 36,33 & 25,31 & 6,94 & 27,73 \\ 0,0 & 5,99 & 3,05 & 13,18 & 23,71 \\ 43,16 & 16,28 & 9,06 & 41,01 & 7,95\end{array}$

L'application à ces données des relations précédentes donne :

$$
\mathrm{D}=0,0044923 \text { soit } 4,49 \text { individus } \cdot \mathrm{ha}^{-1}
$$

Par rapport à la densité vraie de 5,04, l'erreur relative est de 11 p. 100, ce qui est assez faible en regard des erreurs relevées d'après l'application de la méthode de Curtis (4).

Signalons également qu'à partir du même type d'observation, Chessel $(2,3)$ a proposé un mode d'interprétation différent conduisant à caractériser le mode de distribution, contagieux on non, dans les populations végétales - notamment steppiques mais il n'allait pas jusqu'à la densité nécessaire à l'estimation des potentialités pastorales. 


\section{CONCLUSION}

La méthode de Crain, plus simple qu'elle ne parait, permet les mesures de densité dont les pastoralistes font usage, avec un biais relativement faible et compatible avec les autres incertitudes inhérentes à l'évaluation de la ressource constituée par les ligneux fourragers des savanes sèches.

\section{BIBLIO G RAPHIE}

1. BURNHAM K., ANDERSON D., 1976. Mathematical models for non parametric inferences from line transect data. Biometrics, 32: 325-336.

2. CHESSEL D. L'échantillonnage d'une population d'individus dénombrables. Université $C$. Bernard, dép. de Biologie générale et appliquée, Lyon, France, $23 \mathrm{p}$.

\section{Summary}

Daget $\mathbf{P}$., Ickowicz A. Evaluation of the density of the ligneous by distance-methods? An estimator

The authors take up again an estimation mean of the ligneous density in savannah from observations close to "Plotless" method ones and show its functioning and applicability.

Key words: Pastures - Measurement - M ethods - Sampling Vegetation - Density - Woody plants - Tropical zones.
3. CHESSEL D., 1973. Mesure de la contagion vraie en échantillonnage par carrés dans l'analyse des populations végétales. Comptes-rendus Acad. Sci., Paris, France, 277 : 1483-1486.

4. CLARK P. EVANS F., 1954. Distance to nearest neighbour as a measure of spatial relationships in populations. Ecol., 35: 445-455.

5. CRAIN B., BURNHAM K., AN DERSON D., LAAKE J., 1978. A Fourier series estimator of population density for line transect sampling. Ville : Salt Lake City, UT, USA, U tah State U niversity Press, $25 \mathrm{p}$.

6. DAGET Ph., ICKOWICZ A., MBAYE M., 1999. Evaluer la densité des ligneux par la méthode des distances ? Un problème. Revue Elev. M éd. vét. Pays trop., 52 : 263-266

7. EMLEN J., 1971. Population densities of birds derived from transect counts. The auks, 88: 323-342.

8. GATES C., MARSHALL W., OLSON D., 1968. Line transect method of estimating grouse population densities. Biometrics, 24: 135-145.

9. VAN WAGNER C., 1968. The line intersect method in forest fue sampling. Forest Sci., 14: 20-26.

Reçu le 13.7.99, accepté le 24.2.00

\section{Resumen}

Daget P., Ickowicz A. ¿Evaluar la densidad de los leñosos por el método de las distancias? U n estimador

Los autores toman un estimador de la densidad a partir de observaciones próximas a las "Plotless methods », demostrando el funcionamiento y la aplicabilidad en caso de los leñosos de las sabanas.

Palabras clave: Pastizales - Medición - M étodos - Muestreo Vegetación - D ensidad - Plantas leñosas - Zona tropical. 